\title{
Application of Linear Network Analysis to Feynman Diagrams
}

\author{
Jon Mathews \\ California Institute of Technology, Pasadena, California
}

(Received September 2, 1958)

\begin{abstract}
An analogy between a linear passive electrical network and a Feynman diagram is pointed out. The power dissipated in the network is closely related to the denominator of the Feynman matrix element, and this similarity provides a very simple proof of Nambu's results concerning the location of the singularities of Green's functions.
\end{abstract}

$\mathrm{R}^{\mathrm{E}}$ ECENTLY Nambu ${ }^{1,2}$ has analyzed in considerable detail the spectral representation of Green's functions, by explicitly using the Feynman diagram form of perturbation theory. The diagrams were considered in configuration space, each $S_{F}$ or $D_{F}$ function was written as an integral over a parameter, and the integrals over internal coordinates were done. Then, by a Fourier transformation of the results, the more familiar type of representation was obtained. By studying the denominator in the resulting integrand, and in particular locating the range of the external momenta for which this denominator vanishes within the range of integration, one can locate branch-point singularities of the Green's function.

It is the purpose of this note to point out that the conclusions reached by Nambu can be reached somewhat more easily by studying Feynman diagrams in momentum space $a b$ initio. An analogy with electrical circuit theory appears, and the results follow immediately from some well-known network theorems.

For definiteness, consider the vertex diagram of Fig. 1. The "energy denominator" of the matrix ele-

FIG. 1. Simple electromagnetic vertex diagram.

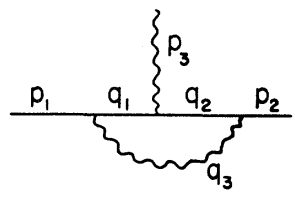

ment is $\left(q_{1}^{2}-M^{2}\right)\left(q_{2}^{2}-M^{2}\right) q_{3}^{2}$, and we must integrate over the virtual momenta $q_{i}$. Rather than do these integrations, we note that integration of a denominator of the form $\left(q^{2}-2 q \cdot A+B\right)^{n}$ over all $d^{4} q$ produces a denominator $\left(B-A^{2}\right)^{m}$. Since $B-A^{2}$ is simply the minimum of $q^{2}-2 q \cdot A+B$, if $q$ is considered a real scalar variable, we shall minimize with respect to all virtual momenta, rather than integrate over them.

As usual, before doing this integration, or minimization, we shall combine the factors of the denominator into one single factor (raised to some power) by using

1 Y. Nambu, Nuovo cimento 6, 1064 (1957).

${ }^{2} \mathrm{Y}$. Nambu (to be published).
Feynman's method of combining denominators; the denominator of Fig. 1 now becomes

$$
\left[a_{1}\left(q_{1}^{2}-M^{2}\right)+a_{2}\left(q_{2}^{2}-M^{2}\right)+a_{3} q_{3}^{2}\right]^{3} .
$$

We must integrate over the $a_{i}$ subject to the conditions $\sum_{i} a_{i}=1, a_{i}>0$.

We shall write the quantity within square brackets in (1) as $F-G M^{2}$, and consider $F$ alone for a moment. It equals the rate of generation of heat that would occur in a network of the form of Fig. 1, with a resistance $a_{i}$ in the branch carrying current $q_{i}$. The conservation of momentum at each vertex of the Feynman diagram is interpreted as the conservation of current at each junction. Now it can be shown ${ }^{3}$ that the current in such a network distributes itself so as to minimize the generation of heat. Therefore, after integration over all internal variables $q_{i}, F$ becomes the actual rate of heating in the network of Fig. 1, with external currents $p_{i}$.

Theorem 4 of reference 1 now follows immediately. For example, if we insert another photon line into Fig. 1, giving the diagram of Fig. 2, and write the new

Frg. 2. Diagram resulting from adding a photon line to Fig. 1 .

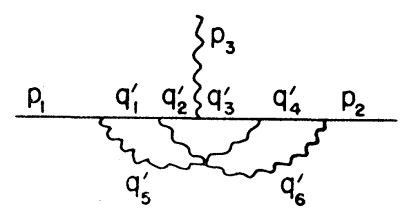

denominator in the form $F^{1}-G^{1} M^{2}$, then for any choice of the parameters $a_{i}{ }^{1}$ in $F^{1}$, and $G^{1}$, there exists a set $a_{i}$ such that

$$
F^{1} / G^{1}<F / G
$$

for arbitrary real scalar $p_{i}$. This is seen as follows: Choose the parameters so that $G=G^{1}$. (This requires $a_{1}{ }^{1}+a_{2}{ }^{1}=a_{1}, a_{3}{ }^{1}+a_{4}{ }^{1}=a_{2}$.) Now it is clear that $F^{1}<F$, by the theorem from circuit analysis ${ }^{3}$ : If the resistance of any branch is increased (decreased), then the resistance of the entire network is increased (decreased).

${ }^{3}$ W. R. Smythe, Static and Dynamic Electricity (McGraw-Hill Book Company, Inc., New York, 1950), p. 233. 